\title{
Tangence
}

\section{Les archives du vent (3)}

Jacinthe Martel, Jacques Paquin, Mariloue Sainte-Marie et Isabelle Kirouac-Massicotte

Numéro 95, hiver 2011

URI : https://id.erudit.org/iderudit/1004048ar

DOI : https://doi.org/10.7202/1004048ar

Aller au sommaire du numéro

Éditeur(s)

Tangence

ISSN

1189-4563 (imprimé)

1710-0305 (numérique)

Découvrir la revue

Citer ce document

Martel, J., Paquin, J., Sainte-Marie, M. \& Kirouac-Massicotte, I. (2011). Les

archives du vent (3). Tangence, (95), 81-99. https://doi.org/10.7202/1004048ar d'utilisation que vous pouvez consulter en ligne.

https://apropos.erudit.org/fr/usagers/politique-dutilisation/ 


\title{
Les archives du vent (3)
}

\author{
Jacinthe Martel, Université du Québec à Montréal, \\ et collaborateurs
}

Trois années seulement se sont écoulées depuis la publication de la première chronique «Les archives du vent ${ }^{1}$ » et $j$ 'ai pourtant l'impression que les choses ont sensiblement changé et que le paysage s'assombrit. En effet, malgré le caractère dynamique de la recherche, dont témoignent notamment les publications, les colloques et la création de nouvelles collections, l'accès aux fonds d'archives se complique et les équipes de recherche consacrées à des objets touchant de près ou de loin le patrimoine littéraire et les archives d'écrivains se font vraisemblablement plus rares.

Les archives des écrivains acquises par BAnQ (Bibliothèque et Archives nationales du Québec) et BAC (Bibliothèque et Archives Canada) exigent souvent, en raison de leur diversité et de leur ampleur, un traitement important qui conduit dans la plupart des cas à la mise au point d'un inventaire ${ }^{2}$ qui constitue pour le chercheur un instrument indispensable, puisqu'il s'agit de son premier et principal accès aux fonds. Mais entre l'acquisition, qui suppose parfois un processus assez long, et le traitement d'un fonds, qui conduit à son entrée dans la banque de données Pistard ${ }^{3}$, il peut s'écouler une année, voire davantage. C'est du moins ce que permet de constater la liste des acquisitions (2008-2009 et 2009-2010 ${ }^{4}$ )

1. Cette chronique s'inscrit dans le cadre du projet «Traces et tracés de l'écriture dans les archives des écrivains» (Jacinthe Martel, Yves Jubinville et Jacques Paquin, CRSH 2009-2012).

2. Malheureusement, plusieurs inventaires sont encore disponibles en format papier seulement et dans une version souvent ancienne. Consacré au fonds Gaston Miron et établi par France Ouellet (sous la supervision de Michel Biron), le dernier répertoire numérique a été publié en 2004.

3. Programme informatisé servant au traitement des archives et à la recherche documentaire disponible en ligne; pour une description de Pistard, voir le site de BAnQ.

4. Outre cette liste, disponible sur le site de BAnQ, nos principales sources pour la rubrique "Archives d'écrivains: nouvelles acquisitions et ajouts" sont: Pistard, $\check{A}$ rayons ouverts ainsi que les sites de BAC et de BAnQ. Prière de me communiquer toutes les informations qui permettraient d'enrichir cette chronique et, en particulier, la bibliographie: martel.jacinthe@uqam.ca. 
diffusée par BAnQ; plusieurs des fonds ou ajouts sont encore «en traitement» et ne figurent pas dans Pistard. C'est dire assez que la recherche, dont l'un des principaux objectifs consiste à mettre en valeur les archives des écrivains, peut négliger des pans entiers d'un fonds parce que plusieurs pièces (carnets, correspondance, etc.), pourtant en cours d'acquisition, ne sont signalées nulle part. En outre, la diffusion des travaux peut accuser un important retard si les documents exigent un trop long traitement. Ce sont là des obstacles difficiles à contrer compte tenu des conditions qui définissent par exemple les politiques d'acquisition et de diffusion des archives privées. L'absence d'articles portant sur les fonds d'écrivains dans les deux premiers numéros de la Revue de Bibliothèque et Archives nationales $d u$ Québec ${ }^{5}$ témoigne également de cet état de fait qui finira sans aucun doute par compromettre la recherche et qui risque fort, à plus ou moins long terme, d'éroder la ténacité de ceux et celles (étudiants ou chercheurs, jeunes ou non) qui s'obstinent, mais pour combien de temps encore, à mener ce type de travaux réalisés le plus souvent dans la solitude d'une bibliothèque. Il reste à souhaiter que cette situation soit temporaire.

\section{Échos}

Heureusement, les expositions consacrées aux archives des écrivains permettent de donner largement accès à des collections ou à des fonds souvent méconnus, d'entrer dans l'atelier des écrivains en mettant au jour des documents inédits et, ainsi, de piquer la curiosité du public et des chercheurs. Il en va ainsi de l'exposition présentée à $B A n Q$, «Roland Giguère. Artisan du rêve ${ }^{6}$ ", qui comprenait de très nombreux documents issus pour l'essentiel du fonds conservé par l'institution (manuscrits, ouvrages, car nets, etc.) et qui étaient répartis selon une structure en trois volets illustrant magnifiquement les différents aspects du travail créateur de Giguère. Étonnamment, l'exposition «Contre-culture: manifestes et manifestations», actuellement présentée à BAnQ et qui

5. Le premier numéro contient cependant, en ce qui a trait aux archives privées, un compte rendu de l'exposition "L'archipel poétique de Paul-Marie Lapointe"(BAnQ, 21 octobre 2008-31 mai 2009) signé Mariloue SainteMarie. À ce propos, voir «Les archives du vent (2)», Tangence, Rimouski et Trois-Rivières, $\mathrm{n}^{\circ}$ 91, automne 2009, p. 113-136.

6. L'exposition a été présentée dans le cadre de la série "Ateliers d'écrivains »; la conférence de Mariloue Sainte-Marie est signalée dans la section "Pistes bibliographiques». Malheureusement, aucun catalogue n’a été publié. 
propose de nombreuses pièces issues de plusieurs fonds et de la collection nationale, contient surtout des imprimés, et un seul manuscrit: un carnet de Patrick Straram. Pourtant, le fonds Josée Yvon contient par exemple un grand nombre de documents variés, notamment des cahiers et des carnets de rédaction, qui révèlent l'ampleur de son travail au moment où elle entreprend un recueil; il aurait été intéressant d'insérer dans l'exposition quelques pièces de son «atelier».

Selon une formule et une disposition très originales, l'exposition "Hubert Aquin et les médias", présentée du $1^{\text {er }}$ au 29 no vembre dernier, a proposé un très grand nombre de documents, peu connus du public en général et des chercheurs en particulier, appartenant principalement aux collections audiovisuelles de la Médiathèque J.-A. DeSève de la Bibliothèque des lettres et sciences humaines de l'Université de Montréal. Outre de nombreuses photos, de la correspondance et des extraits de textes, l'exposition offrait aux visiteurs la possibilité d'écouter ou de visionner des entrevues avec Hubert Aquin ou conduites par lui, des émissions radiophoniques et des œuvres de création, ainsi que des films réalisés par l'ONF auxquels il a collaboré à divers titres ${ }^{7}$.

Le programme d'Ateliers-conférences proposé par BAnQ ${ }^{8}$ peut également constituer un outil de diffusion précieux, notamment sur le plan pédagogique. Ainsi, l'atelier «Les archives culturelles: la fabrique de l'art », coanimé par Hélène Charbonneau, archiviste au Centre d'archives de Montréal, et Mariloue SainteMarie, agente de recherche à la Direction de la recherche et de l'édition, comprend deux volets complémentaires qui visent la découverte de la collection des archives privées: le premier aborde la question de l'accès aux fonds et présente les différents outils de recherche (inventaires, Pistard, etc.) disponibles, tandis que le second présente des documents issus de divers fonds d'artistes et d'écrivains (manuscrits, dessins, carnets, etc.) qui sont notamment présentés du point de vue de la genèse des œuvres.

Enfin, il importe de souligner l'ouverture, l'automne dernier, du Musée de la Médiathèque littéraire Gaëtan Dostie qui propose un ensemble étonnant de livres, d'œuvres, de peintures, de

7. L'exposition, qui comportait trois volets (radio, cinéma, télévision), coïncidait avec la mise en ligne de la collection d'objets numériques «Calypso » de l'Université de Montréal (http://calypso.bib.umontreal.ca/).

8. Cet atelier a été créé en janvier 2011. Pour une présentation complète du programme, voir le site de BAnQ. 
manuscrits, etc. acquis par le collectionneur au fil des ans ${ }^{9}$, ainsi que la création du Prix Jacques Cotnam décerné par la collection "L'archive littéraire au Québec», dirigée par Bernard Andrès aux Presses de l'Université Laval, et qui s'adresse aux étudiants de mâ̂trise et de doctorat dont les travaux "problématisent les notions de l'archive, des sources et de la mémoire dans une perspective littéraire et historique ${ }^{10} »$. En s'ouvrant à une diversité de sujets et de corpus, ce prix, qui sera décerné pour la première fois en avril 2012, s'ajoute fort utilement au Programme de soutien à la recherche de BAnQ qui s'adresse aux étudiants (maîtrise ou doctorat) et aux chercheurs dont les travaux portent sur ses collections.

$\star * *$

Cette chronique se clôt sur des «Pistes bibliographiques», qui offrent une mise à jour des recensions déjà signalées; elles sont précédées des «Notes de recherche» de Jacques Paquin, qui présente un aperçu des travaux amorcés avec son équipe dans le fonds Gatien-Lapointe conservé à Trois-Rivières. À la fois riches et complexes, les archives du poète n'avaient encore fait l'objet ni d'une enquête systématique ni d'un inventaire descriptif complet permettant d'en évaluer précisément la portée et, surtout, d'en comprendre à la fois le fonctionnement et les enjeux pour la lecture de l'œuvre. J. Paquin soulève quelques-unes des questions qui ont surgi au cours de sa recherche ${ }^{11}$. Par ailleurs, dans la thèse de doctorat dont elle livre ici le résumé, Mariloue Sainte-Marie propose une édition critique d'une partie importante de la correspondance de Gaston Miron (1949-1965) qui enrichit de façon déterminante notre connaissance de l'œuvre; la riche documentation utilisée par M. Sainte-Marie permet par exemple de restituer aux lettres toute leur pertinence en regard notamment des documents d'archives dont la publication a été amorcée en 2003 avec Poèmes épars ${ }^{12}$.

15 mai 2011

9. Pour une description de la collection, voir le site de la Médiathèque : http://algi.qc.ca/asso/mlgd/depart/html.

10. Pour une description complète du Prix, voir la section consacrée à la collection "L'archive littéraire au Québec» sur le site des PUL.

11. Les articles et communications portant sur le fonds Gatien-Lapointe sont signalés dans la rubrique bibliographique. Voir aussi «Les archives du vent (2)», art. cité.

12. Gaston Miron, Poèmes épars, éd. Marie-Andrée Beaudet et Pierre Nepveu, présentation de Pierre Nepveu, Montréal, l’Hexagone, coll. «L'appel des mots», 2003. 


\section{Notes de recherche}

\section{Le fonds Gatien-Lapointe: la place de la prose}

Jacques Paquin, Université du Québec à Trois-Rivières

En 1994, la succession Gatien Lapointe a cédé les archives personnelles du poète à Trois-Rivières, au Musée des arts et traditions populaires, devenu en 2003 le Musée québécois de culture populaire. Le fonds légué par les héritiers est composé d'un classeur à deux tiroirs et de vingt-cinq cartons, et il couvre la seconde moitié des années 1950 jusqu'en 1983, année du décès de Gatien Lapointe. Le musée a créé un répertoire sommaire qui donne un aperçu du contenu des archives, dont l'étendue est évaluée à 4,80 mètres linéaires. Quant au classement préexistant, on présume qu'il est de Lapointe, mais pas exclusivement; en effet, certains dossiers portent les initiales B.G., qui renvoient vraisemblablement à Bernadette Guilmette ${ }^{13}$. Le musée n'a attribué à ce jour aucune cote de classification au fonds et le critère de description qui a prévalu est l'"article», compte tenu des intitulés inscrits sur les chemises. On a cependant indiqué le nombre de pièces contenues dans chaque dossier. L'ensemble du fonds reflète les trois grands foyers d'activité de Gatien Lapointe: le poète (1953-1983), le professeur (1962-1983) et l'éditeur (1971-1983). Le classeur regroupe exclusivement des écrits épistolaires: lettres aux membres de la famille, à des poètes canadiens ou étrangers et à des auteurs associés aux Écrits des Forges ${ }^{14}$. On y trouve enfin une correspondance administrative qui signale des ententes avec divers agents culturels (comités de revue et éditeurs) ou encore qui concerne les conditions d'embauche à titre de professeur, notamment au sein des deux principales institutions fréquentées par le poète: le Collège militaire royal de Saint-Jean (à Saint-Jean-sur-Richelieu) et l'Université du Québec à Trois-Rivières. Le contenu des cartons se partage entre des recueils ou des poèmes sous forme manuscrite ou dactylographiée; on trouve également des carnets de notes contenant des réflexions éparses et des notes de cours, des articles, pré-

13. B. Guilmette est l'un des deux donateurs d'un fonds sur lequel je reviendrai plus loin.

14. Gatien Lapointe a étudié à Paris et séjourné en France (1956-1962), où il a développé un réseau littéraire. À son retour au Québec, il a enseigné pendant six ans au Collège militaire royal de Saint-Jean-sur-Richelieu avant de devenir professeur à l'Université du Québec à Trois-Rivières, où il a fondé les Éditions des Forges en 1971 (renommées Écrits des Forges en 1973), avec l'aide de quatre étudiants, dont B. Guilmette. Il a dirigé cette maison de poésie jusqu'à sa mort en 1983. 
faces ou conférences, publiés ou à l'état d'ébauche, ainsi que de nombreuses photocopies d'articles ayant servi à la préparation des cours ou de comptes rendus critiques de son œuvre, etc. Le répertoire dénombre aussi de nombreuses photographies ainsi que des affiches de récitals et de poèmes. Parmi les pièces maîtresses du fonds, on a inventorié: un journal intime de cent dix pages manuscrites, que le poète a rédigé de 1950 à 1956, des centaines de poèmes inédits ${ }^{15}$, dont un grand nombre sont corrigés ou annotés, et une correspondance tenue pendant le séjour de Lapointe en France, de 1956 à 1962, époque qui coïncide avec sa reconnaissance comme poète par la critique française.

Il existe un autre fonds remis par le couple Bernadette et Armand Guilmette avec lequel Lapointe a entretenu toute sa vie des liens d'amitié. Ce fonds, antérieur à celui déposé par les héritiers du poète, est constitué de 88 cartons qui n'ont pas encore été inventoriés. Toutefois, un coup d'œil d'ensemble permet d'identifier des articles promotionnels des Forges, des curriculums vitae, des copies de recueils, dont certains annotés, des travaux d'étudiants, les notes manuscrites de cours suivis à l'Université de Montréal de même que celles engrangées en vue d'une thèse de doctorat à Paris. Les Guilmette ont aussi cédé l'imposante bibliothèque personnelle du poète ainsi que des meubles et des objets lui ayant appartenu ${ }^{16}$. Ma recherche porte en particulier sur l'étude des proses de Lapointe, ce qui inclut: le journal, la correspondance et les notes éparses. La première étape a consisté à dresser un inventaire détaillé du fonds déposé par la Succession Lapointe, dans le but précisément d'évaluer la nature et l'importance des documents, qu'ils relèvent ou non du genre poétique. Afin de décrire chaque feuillet ou groupe de feuillets, une fiche contenant cinq rubriques a été élaborée; elle précise:

- le titre du document, qui est reproduit tel quel si le document porte effectivement un intitulé (sinon l'incipit est signalé entre crochets);

15. Le journal fait partie de l'inventaire du Musée, mais il a fallu dépouiller le fonds pour identifier les textes inédits. Ce travail a été réalisé avec l'aide de trois étudiantes (Andrée-Anne Fréchette, Marie-Pier Laforge-Bourret et Maria Vélez) dans le cadre d'un projet de recherche subventionné intitulé «Traces et tracés de l'écriture dans les archives d'écrivains» (Yves Jubinville, Jacinthe Martel et Jacques Paquin, CRSH, 2009-2012).

16. À titre d'exemple: une petite table, ses lunettes, des photos, son passeport, etc. 
- la datation, précise ou estimée. Comme Lapointe réutilisait fréquemment le verso des procès-verbaux des réunions du département de français de l'UQTR, nous pouvons, pour la période 1969-1983, identifier sinon la date, du moins la période à laquelle il a rédigé le texte;

- le nombre de pages contenues dans le document;

- le support matériel: outre les procès-verbaux, Lapointe a eu recours à quelques reprises à des napperons de restaurant et même à un paquet de cigarettes;

— les caractéristiques de l' "écriture». Cette rubrique permet d'indiquer si la feuille est remplie au recto ou au verso, si l'écriture est manuscrite (et, s'il y a lieu, la couleur de l'encre), s'il s'agit d'un dactylogramme ou d'une photocopie. C'est également sous cette rubrique que nous donnons un aperçu du contenu du document, c'est-à-dire le type de texte auquel il appartient (réflexions, note de cours, vers, prose poétique, etc.). La présence de ratures et d'ajouts est aussi consignée. Nous avons d'emblée indiqué s'il s'agissait de poèmes ou de fragments de poème afin de repérer les inédits.

En voici un exemple:

Titre: Ce soir-là, la troupe du Pilobolus.../Corps et graphies.

Date: probablement après 1971.

Page: 1

Support: feuille lignée au verso.

Écriture: Au verso, la feuille est lignée mais sans écriture. Recto: Poèmes dactylographiés, numérotés de 1 à 3 de Corps et graphies.

En marge des poèmes: écriture manuscrite, encre noire, "Voici donc mon je...", notes appréciatives sur l'écriture dans la marge de gauche, notes sur Pilobolus Dance Theatre en haut de la feuille; ajouts et ratures à la main sur les textes en marge et sur les poèmes.

* Poésie 


\section{Figure 1. Fac-similé de Corps et graphies}

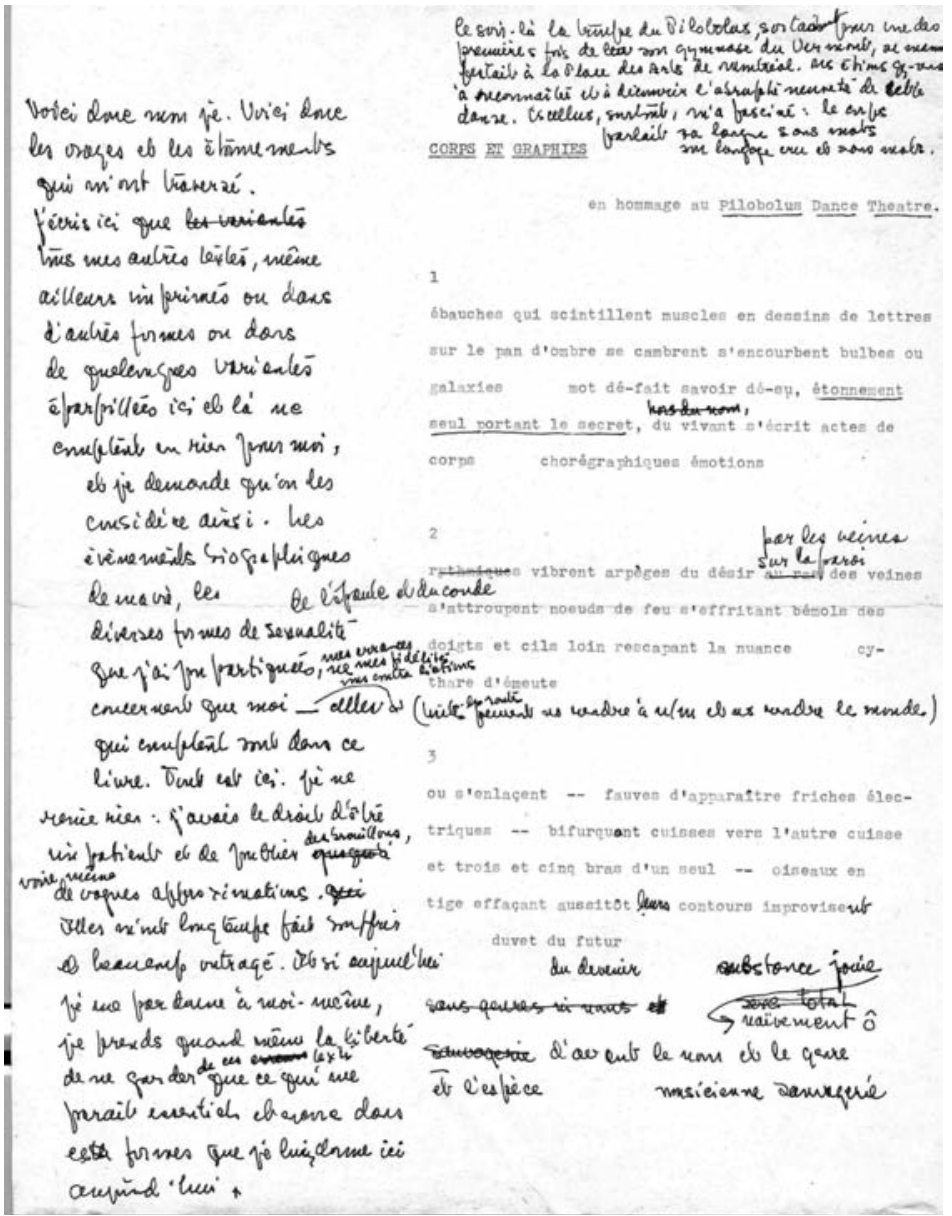

Étant donné la richesse et la complexité de ce fac-similé de Corps et graphies (figure I), je me limiterai aux textes en prose et, en particulier, à celui qui apparaît dans la marge de gauche, que je reproduis ici, dans une transcription linéarisée:

Voici donc mon je. Voici donc les orages et les étonnements qui m'ont traversé. J'écris ici que tous mes autres textes, même ailleurs imprimés ou dans d'autres formes ou dans de quelconques variantes éparpillées ici et là ne comptent en 
rien pour moi, et je demande qu'on les considère ainsi. Les événements biographiques de ma vie, les diverses formes de sexualité que j'ai pu pratiquées, $[s i c]<$ mes errances, mes fidélités mes contradictions $>$ ne concernent que moi (toutes les routes peuvent ns rendre à $\mathrm{n} / \mathrm{m}$ et nous rendre le monde) - celles qui comptent sont dans ce livre. Tout est ici. Je ne renie rien: j'avais le droit d'être impatient et de publier des brouillons, < voire même $>$ de vagues approximations. Elles m'ont longtemps fait souffrir et beaucoup outragé. Et si aujourd'hui je me pardonne à moi-même, je prends quand même la liberté de ne garder $<$ de ces textes $>$ que ce qui me parait essentiel et encore dans les formes que je lui donne ici aujourd'hui ${ }^{17}$.

L'intérêt de ce texte réside dans la polarisation entre l'écriture du poème et l'écriture en prose, qui occupe la marge du texte devant être publié. L'épigraphe qui apparaît sous «Corps et graphies» se lit comme suit, avec des mots soulignés à l'encre: "en hommage au Pilobolus Dance Theatre». Mais dans la version finale, le commentaire en prose (haut de la page) ne subsistera pas et le texte en exergue sera en grande partie modifié: «En marge d"“Ocellus" du PILOBOLUS DANCE THEATRE, le $1^{\text {er }}$ février 1977 à Montréal». Le contenu de la prose renvoie à la satisfaction du poète d'avoir exprimé dans ce projet de recueil, la part la plus authentique de son Moi, qu'il oppose à tout ce qu'on pourrait lire de lui, aussi bien dans ses recueils antérieurs que dans ses archives personnelles. Non seulement Lapointe y affiche la supériorité de l'expression poétique sur l'anecdote autobiographique, mais il considère ce dernier recueil comme le plus achevé de tous ${ }^{18}$. Le texte en prose est postérieur au poème et pourrait avoir eu une visée préfacielle à l'origine. Il ressemble aussi à un testament ou à un avertissement de la part du scripteur à l'endroit de son lecteur éventuel, qu'il invite à ne pas chercher ailleurs que dans les recueils le Moi authentique du poète. Bien que les deux formes de texte occupent leur espace propre, le texte en prose franchit l'espace de la marge pour envahir le blanc typographique du texte poétique, à un point tel qu'il faut relire attentivement ce fragment pour l'attribuer au texte en prose. En effet, sur le plan visuel, ce débordement suscite une confusion avec les annotations ayant le poème comme objet.

17. Les chevrons indiquent tout ce qui est en interligne; les ratures sont reproduites telles quelles.

18. Gatien Lapointe, Corps et graphies, Trois-Rivières, Éditions du Sextant, 1981, s. p. Réédition: Corps et graphies [et autres textes], Trois-Rivières, Écrits des Forges, 1999, p. 43-62. 
De la même manière, on remarque qu'une autre annotation de la même encre, celle-là liée au texte poétique, occupe une partie de la marge réservée au texte en prose ("de l'épaule et du coude»). Alors que le commentaire en marge ne sera pas repris dans la version finale du recueil, le poème donnera lieu à une version finale nettement distincte de la variante. La conjugaison de ces deux formes de texte (le discours en prose et le poème), redoublées par deux empreintes matérielles distinctes (manuscrite et dactylographiée), donne un aperçu de la richesse interprétative suggérée par leur cohabitation au sein d'un même espace. Les archives de Gatien Lapointe révèlent non seulement la place que la prose, plus discrète dans l'œuvre publiée, occupe dans son parcours réflexif, mais aussi l'importance des écrits demeurés à la périphérie de l'œuvre et qui n'en constituent pas moins des traces tangibles d'une poétique et d'une conception de la littérature.

\section{Fiche signalétique}

Mariloue Sainte-Marie, "Écrire "du fond de cette attente éparpillée partout dans la foule”. Édition critique de lettres de Gaston Miron (1949-1965) ", thèse de doctorat, Québec, Université Laval, 2010, 2 vol.

\section{Ce document reprend intégralement le résumé joint à la thèse de doctorat.}

Cette thèse présente une édition critique de lettres qu'a écrites Gaston Miron entre 1949 et 1965. Le corpus comprend deux cent six lettres, pour l'essentiel inédites, se répartissant entre trente-six destinataires. À ce corpus s'ajoutent quatorze ébauches de lettres. Les destinataires de Gaston Miron se répartissent en deux catégories différentes. La première, celle des amis et des écrivains - dont font notamment partie les lettres à Guy Carle, Claude Haeffely, Rina Lasnier, Fernand Ouellette, Henri Pichette, Jean-Guy Pilon, Michel van Schendel —, présente des ensembles de lettres quantitativement plus importants dont le ton est volontiers celui de la confidence. L'épistolier Miron y livre ses doutes sur lui-même et sa poésie, il expose sans pudeur les tâtonnements de sa pensée et débat avec passion de ses idéaux sociaux. Comme il n'a cessé de le faire dans le cadre des entretiens qu'il a donnés tout au long de sa vie ou dans certains textes en prose tel «Un long chemin », Miron réitère, poursuit, peaufine dans ses lettres le récit de son parcours personnel et littéraire. La seconde catégorie 
de destinataires, celle des interlocuteurs occasionnels - tels JeanCharles Falardeau, Claude-Henri Grignon ou André Langevin - , ne donne souvent à lire qu'une ou deux lettres par destinataire. Ponctuelles, ces lettres répondent souvent à une demande ou à un événement précis.

Le corpus de lettres sélectionné souhaite rendre compte tout à la fois de la maturation intellectuelle, politique et poétique de l'écrivain Miron et de l'élaboration de son ouvre. Il comprend les lettres témoignant d'abord de la formation intellectuelle et littéraire du jeune Miron (1949-1953), puis des années d'écriture des grands cycles poétiques (1954-1965) — «La marche à l'amour", «La batèche» et «La vie agonique» — dont il publie des fragments dans des revues et journaux. Ces lettres dressent un tableau cohérent et vivant, une sorte de journal où s'entremêlent politique, littérature et confidences. Tantôt fonctionnelles, tantôt polémiques ou intimes, les lettres de Miron éclairent de manière originale l'engagement et la venue à l'écriture d'un jeune éditeur, intellectuel et poète dans le Québec des années 1950 et 1960.

\section{Pistes bibliographiques}

Isabelle Kirouac-Massicotte, Université du Québec à Montréal

\section{ARCHIVES D'ÉCRIVAINS : NOUVELLES ACQUISITIONS ET AJOUTS}

Bouchard, Michel-Marc (BAC, LMS-0259). Nouveau fonds; en traitement depuis 2008. Documents textuels et sonores.

Buissonneau, Paul (BAnQ, MSS465). Ajout en 2009-2010; 1,3 m. de documents textuels.

Dagenais, Pierre (BAnQ, MSS435). Ajout en 2008; en traitement.

DAIGLE, France (BAC, R12025-0-4-F et LMS-0262). Nouveau fonds acquis en 2004; traité en 2008. 1,94 m. de documents textuels, publiés et inédits (romans, poésie, théâtre, cinéma).

Desjardins, Louise (BAnQ, Centre d'archives de l'AbitibiTémiscamingue et du Nord-du-Québec, P232). Nouveau fonds acquis en 2000. 5,17 m. de documents textuels: romans, correspondance, photographies, poésies, essais et nouvelles.

DuCHARME, Réjean; collection Rosette Rohana (BAC, R12366-0-1F et LMS-0280). Nouveau fonds acquis en 2008. Comprend 12 lettres de Réjean Ducharme datant des années 70 et 80 . Fonds 
fermé à la consultation jusqu'à la mort de R. Ducharme dont le fonds est conservé à BAC (LMS0130).

GRANDBOIS, Alain; voir fonds Lucienne Boucher (BAnQ, P766; en cours de traitement) qui comprend des lettres envoyées par Grandbois entre le 10 septembre 1932 et le 10 octobre $1933^{19}$. Cet ajout n'est pas signalé dans Pistard.

Grignon, Joseph-Jérôme (BAC, R12539-0-7-F et LMS-0261). Nouveau fonds acquis en 2008. $8 \mathrm{~cm}$ de documents textuels composés de conférences, de poèmes, de chansons, de partitions de musique, de monologues et de dialogues écrits pour la scène, de lettres, de notes ainsi que de coupures de journaux.

GroulX, Lionel (BAnQ, CLG1). Nouveau fonds, acquis en 20092010, provenant de la Fondation Lionel Groulx et du Centre de recherche Lionel Groulx. 41,16 m. de correspondance et de manuscrits d'œuvres historiques ou littéraires. Le fonds contient aussi des photographies et des enregistrements sonores.

Guèvremont, Germaine (BAC, R12045-0-0-F et LMS-0260). Nouveau fonds acquis en $2004.1,41 \mathrm{~m}$. de documents textuels comprenant des romans, des nouvelles, des écrits autobiographiques et journalistiques, des conférences, des textes pour la télévision et la radio.

LABERge, Albert (BAnQ, MSS417). Ajout en 2004: $11 \mathrm{~cm}$. Lot acquis de Pearl Laberge.

LAPOINTE, Paul-Marie (BAnQ, MSS47). Nouveau fonds acquis en 2005. Comporte des documents relatifs aux douze recueils publiés entre 1948 et 2004 .

LECLERC, Gilles (BAnQ, Centre d'archives de la Mauricie et du Centre-du-Québec, P130). Nouveau fonds acquis entre janvier et septembre 2009. 5,12 m. de documents textuels, dont des manuscrits d'œuvres inédites ou inachevées et de la correspondance.

Maillet, Andrée (BAnQ, P798). Nouveau fonds acquis en 20092010. 2,8 m. de documents textuels: œuvres écrites entre 1939 et 1977, publiées et inédites, agendas, journaux intimes, entre-

19. Voir aussi Lettres à Lucienne, Montréal, l'Hexagone, 1987. L'avant-propos, l'introduction et de nombreuses notes sont de Lucienne Boucher. 
vues, correspondance (avec Jacques Ferron, André Melançon et Andrée Paradis), etc. Ce fonds n'est pas signalé dans Pistard.

Meunier, Claude (BAnQ, MSS474). Ajout en 2010. 1,07 m. de documents dont plusieurs textes manuscrits écrits entre 1973 et 2005. Ce fonds n'est pas signalé dans Pistard.

ROYER, Jean (BAnQ, MSS442). Ajout fait en 2003 : 6,4 m. de documents textuels.

Saint-Denys Garneau, Hector de ; collection Claude Décarie (BAC, R12405-0-4-F et LMS-0281). Nouveau fonds acquis en 2008. 0,2 cm: deux lettres adressées à Claude Décarie, compagnon d'études de Saint-Denys Garneau au Collège SainteMarie, qui ont été publiées dans l'édition critique des œuvres de Garneau en 1970.

Turcotte, Élise (BAnQ, P852). Nouveau fonds; en traitement depuis $2011.3 \mathrm{~m}$. de documents textuels: manuscrits, carnets, textes et poème inédits, correspondance, etc.

VALOIS, Léonise (BAnQ, P834). Nouveau fonds; en traitement depuis 2010. $0,22 \mathrm{~m}$. de documents textuels: poèmes publiés et inédits, notes manuscrites, journal personnel, correspondance, etc.

Vlb ÉDiTeur (BAnQ, P812). Nouveau fonds; en traitement depuis 2009 ; 1,64 m. de documents textuels; comprend plusieurs dossiers d'auteurs.

\section{Voir aussi, pour le théâtre:}

KiefFer, Daniel (BAnQ, P688). Fonds acquis en 2000; ajouts en 2001, 2004 et 2009. 0,03 m. de documents textuels constitués de photoreportages concernant Trois fois chantera et plusieurs pièces de théâtre: À toi, pour toujours, ta Marie-Lou et Les hauts et les bas d'la vie d'une diva.

LATUliPPe, Gilles (BAnQ, MSS471). Fonds acquis en 2000; ajout en 2009. Documents textuels: œuvres manuscrites ou imprimées (théâtre burlesque, comédies musicales, téléséries et chansons ${ }^{20}$ ).

20. À propos des fonds de Paul Buissonneau, Alain Grandbois, Claude Meunier, Daniel Kieffer et Gilles Latulippe, voir "Coup d'œil sur les acquisitions patrimoniales ", À rayons ouverts, Montréal, n 81, automne 2009, p. 49-50. Pour le fonds Andrée Maillet, voir «Coup d'œil sur les acquisitions patrimoniales », $\check{A}$ rayons ouverts, Montréal, nº 83, printemps-été 2010, p. 47. 
Pelletier, Gilles (BAnQ, P814). Nouveau fonds acquis en 2009. $0,47 \mathrm{~m}$. de documents textuels et audiovisuels, correspondance, textes annotés, etc.

\section{EXPOSITIONS}

«Roland Giguère. Artisan du rêve». BAnQ, série "Ateliers d'écrivains», exposition tenue à la Grande Bibliothèque du $1^{\text {er }}$ décembre 2009 au 9 mai 2010. La commissaire d'exposition, Mariloue Sainte-Marie, a prononcé une conférence intitulée " "Paysage issu de la nuit": Roland Giguère, poète et artiste» à la Grande Bibliothèque le 26 janvier 2010. Disponible en ligne sur le site de BAnQ.

«Hubert Aquin et les médias». Exposition tenue à l'atrium de la Bibliothèque des lettres et sciences humaines de l'Université de Montréal du $1^{\text {er }}$ au 29 novembre 2010.

«Contre-culture: manifestes et manifestations ». BAnQ, série "Ateliers d'écrivains», exposition tenue à la Grande Bibliothèque du 8 février 2011 au 29 janvier 2012. Commissaire: Mariloue Sainte-Marie. Documents tirés des fonds Denis Vanier, Josée Yvon, Patrick Straram et Raoûl Duguay. Une lecture publique, La contre-culture contre quoi?, a eu lieu le 15 février à la Grande Bibliothèque. En complément de l'exposition, une conférence de Mariloue Sainte-Marie, «Contreculture: manifestes et manifestations ", a eu lieu le 3 mars 2011.

\section{ÉDITIONS DE TEXTES ET DE MANUSCRITS}

La Gazette littéraire de Montréal (1778-1779), édition présentée par Nova Doyon, annotée par Jacques Cotnam en collaboration avec Pierre Hébert, Québec, Presses de l'Université Laval, coll. "L'archive littéraire au Québec », 2010, 977 p.

Aubert de Gaspé, Philippe, "Édition du manuscrit partiel des Mémoires de Philippe Aubert de Gaspé», établie par Marc André Bernier, Claude La Charité et Isabelle Lachance, dans Marc André Bernier et Claude La Charité (dir.), Philippe Aubert de Gaspé mémorialiste, Québec, Presses de l'Université Laval, coll. «Cultures québécoises», 2009, p. 163-234. 
Un extrait de cette édition a été publié en 2008: "Transcription d'un extrait du chapitre VII du manuscrit partiel des Mémoires de Philippe Aubert de Gaspé», Tangence, Rimouski et Trois-Rivières, $n^{\circ} 87$, été 2008, p. 102-105.

Roy, Gabrielle, Euvres complètes, édition sous la supervision de François Ricard (avec la collaboration de Jane Everett, Sophie Marcotte, Isabelle Daunais et Dominique Fortier), Montréal, Éditions du Boréal, coll. "Édition du centenaire ${ }^{21}$ ", 12 volumes prévus (2009-2013). Cette collection présente «le texte définitif de tous les livres que Gabrielle Roy considérait comme faisant partie de son œuvre». Le volume XII comprendra Le temps qui m'a manqué, publié pour la première fois dans la collection «Cahiers Gabrielle Roy» aux Éditions du Boréal en 1997.

Déjà parus: Bonheur d'occasion, vol. I; La petite poule d'eau, vol. II; Alexandre Chenevert, vol. III; Rue Deschambault, vol. IV.

Roy, Gabrielle, "Les Vacances ", édition établie par Sophie Marcotte, Annick Lavogiez et François Ricard, dans Gabrielle Roy et l'art du roman, Montréal, Éditions du Boréal, coll. «Cahiers Gabrielle Roy», 2010, p. 293-328.

\section{COLLOQUES ET COMMUNICATIONS ${ }^{22}$}

Colloque international «Parcours de génétique théâtrale. De l'atelier d'écriture à la scène », Université de Lisbonne, 9-11 décembre 2009.

Jubinville, Yves, "Traces et tracés de l'écriture: pour une approche génétique du texte dramatique contemporain ", 11 décembre 2009.

Colloque «Les archives de l'intimité: des journaux intimes aux blogues», Séminaire 2011 du GARM ${ }^{23}$ (Groupe d'Archivistes

21. À propos de cette édition, voir le site des Éditions du Boréal; voir aussi le site http://gabrielleroy.mcgill.ca.

22. Seules les communications portant sur des questions d'archives ou d'éditions sont recensées ici.

23. Le séminaire 2010 du GARM s'intitulait «L'acquisition et le traitement des archives privées numériques: une pratique à définir» (Université Concordia, 23-24 février 2010). Les programmes des séminaires ainsi que plusieurs exposés et conférences sont disponibles sur le site MontréaListes, portail montréalais des archives (http://ville.montreal.qc.ca/montrealistes). 
de la Région de Montréal), Université Concordia, 22-23 février 2011.

Roy, Julie, «Les archives épistolaires", participation à une table ronde, 22 février 2011.

JubinviLle, Yves, "Pour une génétique du texte théâtral avec ou sans manuscrits», colloque international «Du bon et du mauvais usage de la théorie théâtrale», organisé par la Société québécoise d'études théâtrales (SQET), Université d'Ottawa, 28 mai 2010.

Leblanc, Julie, «Pratiques intertextuelles dans les Carnets inédits de Marie-Claire Blais », Séminaire général 2008-2009 de l'ITEM, ENS (Paris), 13 décembre 2008.

MarcotTe, Sophie, "The HyperRoy Project», colloque international «Conference on Editorial Problems», organisé par Editing Modernism in Canada, Université de Toronto, 24 octobre 2010.

MARcotTe, Sophie, «Recherche actuelle sur Gabrielle Roy», colloque international, Congrès bisannuel de l'American Council for Quebec Studies, Burlington, 5 novembre 2010.

Marcotte, Sophie, "Alexandre Chenevert et The Cashier: chronique d'une réception annoncée», colloque international de l'ACQL/ACQL, Congrès de la Fédération canadienne des sciences humaines, Frédéricton, 28 mai 2011.

Noethlichs, Wolfgang et Danielle Leger, «Patrimoine et mémoire du théâtre au Québec », BAnQ, Grande Bibliothèque, le 9 février 2010. Disponible en format MP3 sur le site de BAnQ.

PAQUin, Jacques, "Le Parcours poétique du journal inédit de Gatien Lapointe (1950-1956) », colloque international «Langages poétiques et poésie francophone en Amérique du Nord», Université York, Toronto, $1^{\text {er }}$ octobre 2009.

PAQUiN, Jacques, «Les archives du corps dans la poésie et les écrits intimes de Gatien Lapointe », colloque international "Américanité, corps et quête de sens en littérature québécoise moderne (1953-1970) », ACFAS, Université de Montréal, 13 mai 2010.

PAQUIN, Jacques, "Silence éditorial et fragments de manuscrits. Radioscopie d'un nouveau commencement chez Gatien Lapointe", colloque international "Comment écrire 
après... ", Congrès de l'APFUCC, Montréal, Université Concordia, 29 mai 2010.

RICARD, François, «Allocution inaugurale: Gabrielle Roy et l'art du roman», "Colloque du centenaire. Gabrielle Roy et l'art du roman»; colloque international, Département de langue et littérature françaises, Université McGill, 21 octobre 2009.

\section{OUVRAGES ${ }^{24}$}

DANDREY, Patrick (dir.), Génétique matérielle, génétique virtuelle. Pour une approche généticienne des textes sans archives, Québec, Presses de l'Université Laval, «Les collections de la République des lettres », 2009, $335 \mathrm{p}^{25}$.

Martel, Jacinthe et Marie Pier Jolicceur, Les carnets d'Alain Grandbois ou l'atelier portatif d'un poète voyageur, Québec, Presses de l'Université Laval, coll. "L'archive littéraire au Québec», 2011, 194 p.

\section{THÈSE}

SAINTE-MARIE, Mariloue, «Écrire "du fond de cette attente éparpillée partout dans la foule". Édition critique des lettres de Gaston Miron (1949-1965) », thèse de doctorat, Québec, Université Laval, 2010, 2 vol.

\section{ARTICLES ET CONTRIBUTIONS À DES OUVRAGES COLLECTIFS}

Bernier, Marc André et Jacinthe Martel, "Les Mémoires de Philippe Aubert de Gaspé: de la genèse d'un texte à la généalogie d'un genre ", Voix et Images, Montréal, vol. 35, $\mathrm{n}^{\circ} 3$ (105), printemps-été 2010, p. 33-49.

Beugnot, Bernard, «Perspectives et prospective. Pour une génétique de l'invention classique», dans Patrick Dandrey (dir.), Génétique matérielle, génétique virtuelle. Pour une approche

24. Voir aussi Michèle Nevert (dir.), Textes de l'internement, Montréal, XYZ éditeur, coll. "Documents», 2009. L'ouvrage contient des textes manuscrits écrits entre 1873 et 1950 par des internés de l'asile Saint-Jean-de-Dieu.

25. Compte rendu de Robert Melançon publié dans Genesis, Paris, Presses de l'Université de Paris-Sorbonne, no 31, 2010, p. 167-168. 
généticienne des textes sans archives, Québec, Presses de l'Université Laval, "Les collections de la République des lettres», 2009, p. 19-29.

Constantin, Danielle, "Sur Lieux où j'ai dormi de Georges Perec", ITEM, http://www.item.ens.fr/index.php?id=76107, mis en ligne le 30 mars 2007. Amalgame des deux articles suivants:

Constantin, Danielle, «Perec et Proust: le travail de la mémoire», dans Claude Filteau et Michel Beniamino (dir.), Mémoire et culture. Actes du colloque international de Limoges (11-12 décembre 2003), Presses universitaires de Limoges, coll. «Fran cophonie», 2006, p. 133-143.

Constantin, Danielle, «Autobiographie vespérale et lieux de sommeil tunisiens", conférence prononcée au colloque international de Tunis (17-20 février 2003); à paraître dans Rabaâ Abdelkefi et Hend Gaha (dir.), La mémoire des lieux dans l'ouvre de Georges Perec. Actes du colloque international de Tunis.

Gauthier, Benoît C., "Conditions d'énonciation scénique: examen des archives d'André Brassard», Tangence, Rimouski et Trois-Rivières, $\mathrm{n}^{\circ}$ 91, automne 2009, p. 116-120.

Groulx, David, "Pistes bibliographiques ", "Les archives du vent», Tangence, Rimouski et Trois-Rivières, $n^{\circ} 87$, été 2008, p. 107-115.

Groulx, David, «Pistes bibliographiques», «Les archives du vent (2) », Tangence, Rimouski et Trois-Rivières, no 91, automne 2009, p. 130-136.

Jubinville, Yves, «La génétique au service de l'histoire », Tangence, Rimouski et Trois-Rivières, nº 87, été 2008, p. 96-102.

Leblanc, Julie, «David Sterne. Pratiques intertextuelles et interdiscursives dans les carnets de Marie-Claire Blais", dans Véronique Montémont et Catherine Viollet (dir.), Le Moi et ses modèles: genèse et transtextualités, Louvain-la-Neuve, Academia-Bruylant, coll. "Au cour des textes», 2009, p. 3952 .

LEBlanc, Julie, «Journal intime, journal d'écriture, journal d'artiste: les carnets inédits de Marie-Claire Blais», dans Monika Boehringer, Kirsty Bell et Hans R. Runte (dir.), Entre textes et images: constructions identitaires en Acadie et au Québec, 
Moncton, Institut d'études acadiennes, coll. «Pascal-Poirier», 2010, p. 345-368. Texte d'une communication présentée au colloque "Se (voir) et savoir: constructions identitaires dans les espaces littéraire et pictural de l'Acadie et du Québec», organisé par l'Association des professeurs des littératures acadienne et québécoise de l'Atlantique (APLAQA), Mount Allison University, Nouveau-Brunswick, 2008.

Marcotte, Sophie, "L'édition électronique de La détresse et l'enchantement de Gabrielle Roy», Tangence, Rimouski et TroisRivières, nº 91, automne 2009, p. 121-129.

VACHON, Stéphane, «Perspectives en génétique balzacienne», dans Kazuhiro Matsuzawa (dir.), Balzac, Flaubert. La genèse de l'œuvre et la question de l'interprétation, Nagoya (Japon), Graduate School of Letters, Nagoya University, 2009, p. 35-45. 